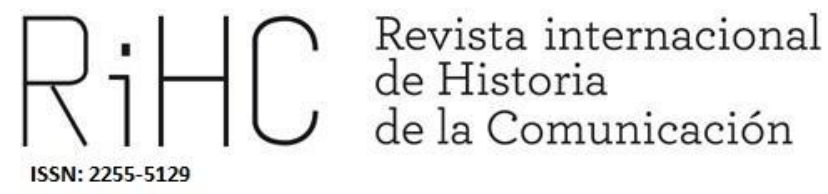

\title{
PERIODISMO Y CAMBIO EN CONTEXTOS DE CRISIS. ANÁLISIS DE LA PRENSA DE BARCELONA DURANTE LA TRANSICIÓN DEMOCRÁTICA (1975-1978)
}

Journalism and change in crisis contexts. Analysis of the Barcelona press during the democratic transition (1975-1978)

DOI: https://dx.doi.org/10.12795/RiHC.2020.i14.13

Recibido: $11 / 04 / 2020$

Aceptado: $10 / 06 / 2020$

Publicado: $15 / 06 / 2020$

Marcel Mauri-Rios

ORCID (i) 0000-0003-2615-8343

Universitat Pompeu Fabra, España

marcel.mauri@upf.edu 
Resumen: La crisis actual que afecta a los medios de comunicación en España comparte muchas características con la que afectó al periodismo después de la muerte de Franco. Durante el siglo XX, el periodismo en España sufrió tres grandes crisis y rupturas: la Guerra Civil de 1936; el inicio de la dictadura; y la transición. Este artículo explora las características que explican la crisis de la prensa entre 1975 y 1978 a partir del caso de la prensa de Barcelona. Una crisis que fue cuádruple -económica (general y de medios de comunicación), de cambio tecnológico e ideológica- y que se estudia a partir de una metodología que combina el análisis histórico, hemerográfico, de contenido y otras metodologías para analizar la estructura económica y corporativa de los medios en esos años claves de la transición democrática.

Palabras clave: Historia, España, periodismo, prensa, crisis

\begin{abstract}
The current crisis affecting the media in Spain shares many characteristics with which it affected journalism after Franco's death. During the twentieth century, journalism in Spain suffered three major crises and ruptures: the Civil War of 1936; the beginning of the dictatorship; and the transition. This article explores the characteristics that explain the crisis of the press between 1975 and 1978 from the case of the Barcelona press. A crisis that was quadruple -economic (general and media), technological and ideological- and that is studied from a methodology that combines historical analysis, hemerographic, content analysis and other methodologies to analyze the economic structure and corporate media in those key years of the democratic transition.
\end{abstract}

Keywords: History, Spain, journalism, press, crisis

\title{
Introducción
}

Desde el año 2008, el sector de la prensa en todo el mundo ha experimentado una situación de crisis múltiple. Un panorama que todavía ha sido más crudo entre el sector comunicativo de España (Almirón, 2009; Casero, 2010; Reig, 2015). A la crisis económica global, se le añade una crisis del sector publicitario (uno de los más resentidos por la situación económica, con caídas que eran del 11\% de media en 2008 pero que seguían cayendo un $8.3 \%$ en 2012 o un 7,1\% en 2016 y, aunque se ralentizaba la caída, en 2017 perdía un 1,4\% respecto el año anterior ${ }^{1}$ ), crisis del modelo de negocio e informativo, y de reconversión tecnológica con la llegada de internet y la web 2.0. En total, esta situación provocó 7.823 despidos solo en los primeros años de crisis (2008-2012) o lo que es lo mismo, una reducción del $15 \%$ de los puestos de trabajo en medios de comunicación; una destrucción de 12.200 puestos de trabajo entre 2008 y 2015²; y un aumento del paro registrado entre periodistas del 74\% entre 2008 y 2016, según datos del Informe Anual de la Profesión Periodística editado por la Asociación de la Prensa de

\footnotetext{
${ }^{1}$ Fuente: Arce Media http://www.arcemedia.es

2 Incluye también, a parte de periodistas, personal administrativo, comercial, trabajadores de rotativos y otros.
} 
Madrid (APM). Este mismo estudio indica que entre 2008 y 2015 han cerrado 375 empresas periodísticas en España. Según el citado informe, "tras la crisis económica, los medios de comunicación españoles se han instalado en un escenario con un nivel de empleo significativamente menor, con unos puestos de trabajo de inferior calidad y con unas relaciones laborales más inestables, factores todos que, necesariamente, van en detrimento de la calidad del trabajo informativo".

Así pues, el sector de la comunicación en España está inmerso, desde 2008, en una crisis que solo tiene parangón con la que el sector sufrió a partir de 1976 (Almirón, 2009: 244). Precisamente, es el final del franquismo donde encontramos una situación de crisis y cambio del sector periodístico que podría ser similar a la actual. Este artículo, que se basa eminentemente en el análisis histórico de la prensa de Barcelona durante la transición democrática en España, estudia a fondo los cambios que sufrió la prensa tras el fin de la dictadura y el inicio de la democracia (1975-1978). También en aquellos años la prensa sufrió una cuádruple crisis: económica (general y de medios de comunicación), de cambio tecnológico e ideológica. Idéntico contexto de crisis, pues, pero con contenido distinto como se explicará en este texto.

\section{Marco teórico y estado de la cuestión}

Si la muerte del dictador Francisco Franco (20 de noviembre de 1975) y los tres años siguientes hasta la llegada de la democracia con la aprobación de la Constitución (6 de diciembre de 1978) marcan un punto y aparte en la historia de España, todo este proceso también conllevará una evolución sin precedentes en la propia historia de los medios de comunicación españoles. Hasta ese momento, y durante el siglo XX, se habían producido tres situaciones de ruptura en la evolución del periodismo. El primero de ellos se produce con el inicio de la Guerra Civil (1936), que ponía fin a una evolución de un siglo de periodismo liberal en España (Guillamet, 2003). El fin del conflicto armado y el inicio del franquismo ${ }^{3}$ representa una segunda ruptura drástica del modelo periodístico hasta ese momento vigente (con lo que supuso de punto final para el periodismo la

\footnotetext{
${ }^{3}$ Guillamet (2003) llama a este periodo de la Guerra Civil dentro de la historia del periodismo como el de la 'Crisis general y quiebra'.
} 
abolición de la libertad de expresión ${ }^{4}$ y la desaparición de la mayoría de rotativos ${ }^{5}$ ). Finalmente, el final de la dictadura provocará una tercera ruptura que, a pesar de tener también aspectos dolorosos (como una nueva desaparición de cabeceras, ahora debido al mercado, como se tratará más adelante), permitirá que la prensa haga su propia transición que la llegará a situar, de nuevo, en los parámetros del periodismo contemporáneo.

El inicio de esta transformación solo se entiende como fenómeno coetáneo y subsidiario (aunque también parcialmente simbiótico) de la propia transición democrática que, a todos los niveles, experimentaba España. Unos cambios que parten de unos antecedentes situados en los últimos años de la dictadura.

La prensa comenzó su propio proceso de cambio unos años antes del inicio de la transición política, a partir de 1966. Ese año las Cortes aprobaron la Ley de Prensa e Imprenta (Ley 14 / 1966, de 18 de marzo de prensa e imprenta), la llamada Ley Fraga, que sustituía la Ley de Prensa de 1938, una legislación que duró casi 30 años y que era absolutamente represiva y basada en principios propios de la Italia de Mussolini (MauriRios, 2010). La ley Fraga no se puede considerar tampoco una ley democrática: prueba de ello es que precisamente durante el mandato de Manuel Fraga Iribarne como ministro de Información y Turismo es cuando proliferan más expedientes y sanciones contra diarios (Barrera, 1995: 154). Sin embargo, y a pesar de que sigue siendo una legislación de prensa típica de una dictadura, supone un ligero avance, un pequeño respiro para los periodistas y los medios y permitirá ir poniendo las bases para comenzar un verdadero proceso de cambio a partir de 1975.

Esta tímida apertura política vendrá motivada por la presión que la propia sociedad española irá ejerciendo: así, en 1973, tres de cada cuatro españoles eran ya partidarios de la libertad de prensa, una exigencia que, sumada a otras voluntades de la ciudadanía como la libertad de culto o de sindicación, obligarán al régimen a mover pieza (Tusell, 2005: 78), aunque sea de forma muy superficial y sin terminar con la represión política.

Desde finales de los sesenta, buena parte de la prensa y de los periodistas -así como otras instituciones hasta entonces afines al franquismo, como la Iglesia o la Universidadirán desligándose ideológicamente del régimen y provocará que el franquismo pierda buena parte del poder del discurso (Casanova, 1994: 27). Pere-Oriol Costa sitúa desde

\footnotetext{
${ }^{4}$ La Guerra Civil y el franquismo suponen la ruptura con casi un siglo de desarrollo del periodismo liberal (Guillamet, 2003: 191). El régimen aprueba una ley de prensa de guerra, en abril de 1938 (nacida con carácter transitorio pero que seguirá vigente hasta el año 1966), inspirada en el fascismo italiano, que define los medios como una institución al servicio del Estado, y deja en manos del gobierno la potestad de decidir qué periódicos se pueden publicar, de nombrar directores, de decidir qué se puede y qué no se puede publicar (censura previa), qué debía publicarse (mediante el envío de consignas), y quién podía ser periodista (Seoane y Sáiz, 2007: 238).

${ }^{5}$ De los 18 diarios que se publicaban en Barcelona al día siguiente del alzamiento militar del 18 de julio, sólo reaparecerán 5 después de la Guerra Civil (Guillamet, 1994: 178).
} 
un poco antes, a partir de 1964 y hasta 1974, el periodo de grandes transformaciones en la prensa, unos cambios que no solo afectan a aspectos de forma o contenido sino que se extienden al rol que los periódicos cumplen como medio de información y como institución política y social (1989: 39).

A partir de la muerte de Franco el espacio comunicativo franquista se agrietará completamente, momento en que los medios, sobre todo la prensa, se verán con la necesidad de emprender un auténtico diálogo social que tendrá como objetivos crear una democrática comunicación política y una verdadera opinión pública (Redero y García, 1991-1992: 110).

Estos antecedentes explican que, una vez se inicie la transición democrática, la evolución vivida por los medios les permitirá estar a punto para iniciar su propio proceso de cambio. En medio de estas transformaciones de índole política, social y económica que experimenta España, e influida por esta misma situación, la prensa también vivirá una transición (o crisis) a todos los niveles: periodístico, ideológico, económico y empresarial.

\section{1 Evolución de la prensa de Barcelona}

El día de la muerte de Franco se publicaban en Barcelona ocho cabeceras de información general: El Diario de Barcelona (1792-1994) -periódico decano de la prensa continental, El Correo Catalán (1876-1985), La Vanguardia española (1881) -que, sin el epíteto de 'española' todavía se publica -, El Noticiero Universal (1888-1985), Solidaridad Nacional (1939-1979) y La Prensa (1941-1979) -publicaciones falangistas, la primera de ellas tomaba el nombre de una antigua publicación anarquista aparecida en Barcelona a principios de siglo XX y que se publicó hasta la guerra civil-, Tele/eXprés (1964-1980) primera publicación privada permitida en Barcelona desde el final de la guerra- y Mundo Diario (1968-1980). En los tres años siguientes, aparecieron tres cabeceras más: Avui (1976-2011) - primera publicación en catalán autorizada desde el final de la guerra civilCatalunya Express (1976-1980) y El Periódico de Catalunya (1978) - que todavía se publica.

La transición que experimentarán los medios catalanes durante estos años se explica también a partir de las características generales apuntadas hasta ahora. La prensa barcelonesa no escapa del contexto político, legal, económico y social general, pero también es cierto que la transición periodística en Cataluña presentará algunas características particulares. En primer lugar, destaca un menor clima de violencia que el que se experimente en Madrid o el País Vasco.

También es muy remarcable la adhesión casi instantánea por parte de toda la prensa (incluso la falangista) a los valores democráticos y a la defensa de las libertades 
colectivas (Guillamet, 2001) por lo que Cataluña no sufrirá el fenómeno de periódicos claramente involucionistas, un hecho que sí sucederá en Madrid donde El Alcázar o el reaparecido El Imparcial se encargarán de intentar torpedear el avance democrático (Redero y García, 1991-1992: 103). Entre la prensa de Barcelona, la voz de los nostálgicos del régimen quedará totalmente silenciada por la mayoría demócrata. Todos los rotativos, desde los de tradición falangista hasta los más próximos a las ideas rupturistas, cerrarán filas en torno a ideas como democracia o libertad. También es cierto que será paradójico, en algunos casos, la conversión a la democracia que vivirán algunos medios. Barrera (1995: 452) introduce el término de 'conversos' para aquellas publicaciones franquistas que, una vez muere Franco, abrazan la democracia y trabajan decididamente para conseguirla.

Finalmente, y como consecuencia de estas características apuntadas, el contexto en el que se desarrolla la prensa de Barcelona durante la transición es en un clima de concordia y entendimiento: en general, los medios catalanes rehúyen las tensiones y disputas entre ellos y se comportarán con una gran madurez para contribuir a fortalecer el proceso democrático. Superadas algunas tensiones entre rotativos vividas en los primeros momentos después de la muerte de Franco, todos los diarios favorecerán la creación de un clima político y social de concordia (Mauri-Rios, 2010). Los tiempos políticamente convulsos y económicamente inestables provocarán que, diez años después del final de la transición democrática, a finales de la década de los 80, de las 11 cabeceras solamente tres resistan: La Vanguardia, Avui y El Periódico.

\section{Objetivos y metodología}

Este artículo se introduce a fondo en los cambios ideológicos, periodísticos, y empresariales que sufrió la prensa española durante la transición democrática a partir de una aproximación metodológica multidisciplinar. El objetivo principal de este estudio es observar y describir la crisis general que el sector periodístico sufrió durante la transición española.

Siguiendo las pautas de autores como Kayser (1974) o Guillamet (2001), y a partir de las propuestas metodológicas de Mauri-Rios (2010), se combina el estudio histórico y hemerográfico y el análisis de contenido con otras metodologías apropiadas para el estudio de la estructura económica y empresarial de las empresas periodísticas. Para poder analizar los cambios sufridos por los medios en esos años de crisis, la investigación toma como objeto de estudio todas las cabeceras de periodicidad diaria, un total de 11 , que se publicaban en la ciudad de Barcelona desde la muerte de Franco hasta la aprobación de la Constitución de 1978: El Diario de Barcelona, El Correo Catalán, La 
Vanguardia española, El Noticiero Universal, Solidaridad Nacional, La Prensa, Tele/eXprés, Mundo Diario, Avui, Catalunya Express y El Periódico de Catalunya.

Tomando como objeto de estudio estas 11 cabeceras, se realiza un estudio pormenorizado del sector periodístico de la capital catalana, a partir del análisis de 4722 piezas periodísticas de los 11 periódicos descritos: 505 editoriales; 896 artículos de opinión; y 3321 noticias, a partir de la elaboración de categorías de análisis específicas para cada género periodístico. Para proceder a la construcción de las categorías para el análisis de contenido, una vez seleccionada la muestra (conformada por estas 4722 piezas o unidades redaccionales), se elaboró una ficha de análisis a partir de las pautas de Kayser (1974), Guillamet (2001) y Mauri-Rios (2010):

00. Número de ficha

0 . Género:

1. Autor:

2. Título:

3. Nombre de la publicación:

4. Fecha de publicación:

5. Páginas:

6. Resumen del contenido:

7. Palabras clave:

8. Materia principal:

9. Materias secundarias:

10. Personas citadas:

11. Instituciones citadas:

12. Descriptores topográficos:

13. Notas:

Finalmente, esta triangulación metodológica incluye un análisis amplio, a partir de documentación y literatura previa, de la situación global del sector en el resto de España que permite describir factores de cambio y crisis.

\section{Análisis}

\subsection{Características de la transición en la prensa}

Esta evolución del periodismo durante la transición democrática irá en paralelo a las modificaciones legales que permitirán acabar con las leyes que consagraban la censura 
y el control político de los medios y recuperar la libertad de prensa. Así las cosas, y a partir del estudio de la prensa de Barcelona, podemos agrupar en cinco grandes bloques las características de este proceso de transición de la prensa entre 1975 y 1978:

- Cambios ideológicos: pluralidad y nuevas cabeceras

- Cambios legales y políticos

- Cambios económicos y empresariales

- Cambios periodísticos

- Cambios de función: agente político

\subsubsection{Cambios ideológicos}

Esta tímida apertura de parte de la prensa de Barcelona (y de buena parte de la prensa española) se irá acentuando a medida que se acerque el final de la dictadura. En este proceso, podemos hablar de dos tipos de periódicos: los que pertenecían directamente al régimen, ya que eran órganos de la cadena del Movimiento Nacional (en el caso de Barcelona se trataba de Solidaridad Nacional y La Prensa); los diarios privados que, aunque no estaban vinculados jurídicamente a la Estado, tampoco disponían de libertad. Es en algunos de estos medios privados donde empezará a vislumbrarse un cierto pluralismo -aunque coartado por las libertades restringidas del momento-, elemento indispensable para comenzar a poner las bases para asumir el rol político protagonista una vez caiga el régimen.

Tampoco hay que olvidar que dentro del bloque de prensa privada no todos los periódicos eran iguales ni se comportaron de la misma forma: así, mientras que había unos medios comprometidos con la democracia desde tiempo antes de la muerte del dictador, otros convivirán con más facilidad con el franquismo y no abrazarán claramente y públicamente la democracia hasta que Franco ya esté muerto. Barrera corrobora este hecho:

También entre la clase periodística tuvo lugar una conversión, inclusive conversión en masa podría decirse, de profesionales de la información que, habiéndose destacado o habiendo defendido los principios y la política del régimen anterior, pasaron a engrosar las filas de los "nuevos demócratas" con mayor o menor fervor, según los casos" (1995b: 452).

A partir de la muerte de Franco se podrá seguir hablando de una división prensa de estado / prensa privada, pero a la vez irá apareciendo una pluralidad ideológica, típica de cualquier sociedad democrática pero inédita hasta este momento en España, que permitirá ver en los quioscos periódicos de diversas tendencias políticas e ideológicas. Al igual que el Estado español comenzó a cambiar de una forma clara y continuada a 
partir de aquel 20 de noviembre de 1975, también la prensa sufrió serias mutaciones. Durante los primeros meses de transición democrática se puede comprobar cómo la aparente similitud ideológica que tenían todos los diarios de Barcelona se empieza a romper. La libertad de prensa que los medios empiezan a arañar a partir de la muerte de Franco (y que se materializará en abril de 1977 con la supresión de los artículos más restrictivos de esta ley) permitirá aflorar el pluralismo que la prensa privada tenía pero que difícilmente podía mostrar, especialmente en terrenos sensibles como la información política o la opinión.

Serán las páginas de los periódicos uno de los primeros espacios en los que los ciudadanos comenzarán a percibir el relativismo de las diferentes ópticas interpretativas que hay en cualquier sociedad plural, una situación que ponía fin definitivamente a la época de las verdades absolutas. Redero y García (1991-1992: 92) apuntan que la peculiaridad de la transición española -pasar de forma legal de una dictadura a una democracia- hará que los medios públicos (como TVE, RNE o la prensa del Movimiento) sigan, al menos durante el primer gobierno de la monarquía, 'esclerotitzados', es decir, al servicio de la propaganda que quisiera emitir el ejecutivo. En estas circunstancias, la prensa escrita independiente aparecerá ante los ciudadanos como la referencia más fidedigna para conseguir información libre.

La apertura y flexibilización del régimen se empieza a notar cuando solo unos meses después del fallecimiento de Franco se autoriza la publicación del primer periódico íntegramente en lengua catalana después del final de la Guerra Civil. Se trata de Avui, que aparece el 23 de abril de 1976 y rompe con 37 años sin prensa en catalán. Unas semanas después aparecerá en Madrid el periódico de referencia de la izquierda, El País, que se convertirá en la cabecera más influyente de la democracia. En diciembre del mismo año sale otro periódico en Barcelona, Catalunya Express, mientras que en octubre de 1978 verá la luz El Periódico de Cataluña, uno de los medios que saldrá más beneficiado de la reestructuración del mercado periodístico que se producirá a finales de la transición. Junto a estos diarios, también es destacable la aparición de un numeroso grupo de revistas - que comenzaron a aflorar a principios de los 70 y durante toda la década - que vendrían a completar este abanico completísimo de publicaciones.

Se puede constatar, pues, que los cambios políticos y la posterior libertad de expresión suscitó una generalizada explosión de optimismo empresarial que hizo aflorar multitud de nuevas publicaciones y convirtió la aparición de nuevas cabeceras en una característica de la transición. Si bien el mercado lector no era demasiado amplio en cifras globales, sí que había una avidez informativa por parte de la población, y los editores querían sacar rédito de esta circunstancia (Redero y García, 1991-1992: 91-92). Todo este movimiento provocará que entre 1976 y 1980 aparezcan 25 diarios nuevos en todo el Estado, algunos con proyectos sólidos detrás que permitirán su consolidación 
como el caso citado de El País, otros con más dificultades estructurales que harán que terminen desapareciendo.

\subsubsection{Cambios legales y políticos}

El estudio de la prensa de Barcelona permite comprobar cómo los medios no estarán inmunes a las consecuencias del cambio político que supone la muerte del dictador y las consiguientes reformas del régimen político que conducen hasta la democratización del Estado. Empiezan a darse las condiciones óptimas para que los periódicos puedan hacer de catalizadores de las reivindicaciones de toda clase que la sociedad exigiría.

Pero no todo son cambios políticos. 0 , al menos, los cambios políticos propician algunas modificaciones legales que permitirán un avance del periodismo. Con la liberalización del Estado también llegará la liberalización de la comunicación pero mientras que la primera responderá a un proceso de reforma, la segunda tendrá algunas características de auténtica ruptura con el pasado. Tanto la radio como la televisión pero sobre todo la prensa privada y pública experimentarán un proceso de ruptura evidente.

\section{Cronología del cambio legal y político:}

El proceso de modificación del marco legal de los medios de comunicación y el desarrollo de la prensa en general experimentan unos momentos claves durante la transición (1975-1978):

- Los medios exploran los límites de la libertad de expresión a partir de la muerte de Franco. Existirá cierta permisividad gubernativa que se debe, en buena parte, a la incapacidad para controlar los medios. Desde la muerte de Franco hasta abril de 1977 encontramos una situación de libertad de prensa de facto ya que sigue vigente aún la Ley Fraga aunque hay una gran permisividad.

- Aparición de nuevos medios: Avui, el primer diario en catalán desde la guerra civil, ve la luz el 23 de abril de 1976 después de recibir el permiso para publicarse en el marco de esta nueva permisividad; en Madrid, aparecen los diarios El País (4 de mayo de 1976) y Diario 16 (18 de octubre de 1976); durante el 1977 en el País Vasco salen un periódico próximo al Partido Nacionalista Vasco (conservador), Deia (8 de junio) y otro vinculado a la izquierda nacionalista, Egin (29 de septiembre).

- Publicación de textos editoriales conjuntos de los principales y mayoritarios periódicos catalanes y españoles: 'Por la unidad de todos', en respuesta a los crímenes de Atocha y a la ola de violencia (enero de 1977), y 'No frustrar una esperanza', tras la legalización del PCE (abril de 1977).

- Supresión del artículo 2 y 69 de la Ley de Prensa e Imprenta de 1966 (Ley Fraga) con la aparición del Real Decreto-Ley sobre Libertad de Expresión (publicado en 
el BOE el 12 de abril de 1977). Este cambio legal es un momento de inflexión muy importante en la transición de la prensa ya que supone la primera medida realmente efectiva que abre el camino a la liberalización del sector y termina con una tradición de casi 40 años de legislación dictatorial. Barrera muestra la importancia que tiene la eliminación de estos artículos:

Por cuanto suprimía el célebre y temido artículo 2 으 de la Ley de Prensa e Imprenta de 1966 que tantos expedientes, sanciones, amenazas, presiones y quebraderos de cabeza había causado a los periodistas. Asimismo se derogaban otras partes de dicha Ley como las referidas a la facultad administrativa para proceder a secuestros y suspensiones de publicaciones (1995b: 454)

Sin embargo, desde algunas instancias y medios de comunicación se le llamará 'Ley antilibelo' y se criticará el hecho de que, a pesar de la apertura que significa la abolición de estos artículos, se mantengan tres limitaciones en cuanto a la libertad de expresión: la unidad de España, la Corona y el ejército.

- Supresión del Ministerio de Información y Turismo (abril de 1977) de donde habían salido leyes como la de Prensa e Imprenta de 1966

- La prensa del Movimiento Nacional pasa a formar parte del ente Medios de Comunicación Social del Estado (MCSE) (1 de abril 1977), debido a la disolución del Movimiento Nacional. Este hecho significará la conversión de la prensa falangista en prensa de Estado democrático y será también el principio del fin de esta cadena de medios de comunicación.

- Libertad de información en la radio (6 de octubre 1977). Se ponía fin a la obligación de las emisoras privadas de radio de conectar cada hora con el informativo de la radio pública y oficialista, Radio Nacional de España (RNE). Se autorizaba que las emisoras privadas pudieran emitir noticiarios propios.

- Configuración del Ente Autónomo de Radio Televisión Española (RTVE, que integraba RNE, Televisión Española y Radio Cadena Española) en el Real Decreto de 28 de octubre de 1977. Esto suponía la creación de la corporación de medios públicos.

- Artículo 20.1 de la Constitución española (aprobada el 6 de diciembre de 1978). Este artículo reconoce y protege los derechos de expresión y de información; suprime la censura previa, y también incluye los derechos a la cláusula de conciencia y al secreto profesional. Con estos derechos constitucionales reconocidos, terminaba la larga travesía en el desierto de los medios de comunicación y los periodistas en poner fin a 40 años de ausencia legal de libertad de prensa, desde la ley de 1938. Los derechos recogidos en la Carta Magna entroncaban con la legislación republicana abolida con el franquismo. 


\subsubsection{Cambios económicos y empresariales}

El paso hacia esta nueva realidad democrática no se hizo exenta de dificultades económicas y empresariales por parte de las propias empresas periodísticas que vieron en muchos casos cómo su influencia política no se traducía en unos beneficios que les permitieran seguir adelante. No hay que perder de vista que las empresas periodísticas son, ante todo, negocios con voluntad (y necesidad) de hacer dinero, a diferencia de los tiempos de la dictadura en la que primaba la función de los periódicos como vía para canalizar la influencia política del régimen por encima de la idea de negocio (Redero y García, 1991/92: 118). Cuando esta forma de entender la función del periodismo cambia con la llegada de las libertades, algo empieza a no funcionar en muchas de estas compañías.

Los datos así lo confirman: entre 1975 y 1984, 60 diarios dejaron de editarse en todo el Estado (25 de estos formaban parte de la Prensa del Movimiento) y los 14 diarios que se publicaban en Barcelona entre 1975 y 1978, a los 10 años solo quedarán tres (La Vanguardia, El Mundo Deportivo y Avui), a los que se sumarán El Periódico de Catalunya ${ }^{6}$ y Sport ${ }^{7}$. Se producía, pues, el proceso a la inversa: si durante los últimos años del franquismo, las ansias de acabar con el régimen habían hecho proliferar las publicaciones (un hecho que aún se vio aumentado con la liberalización de la prensa que llegó con la transición), la llegada de la democracia -con las consiguientes leyes de mercado- supuso la desaparición de buena parte de estos medios.

Estas son las principales dificultades a las que tuvieron que hacer frente los medios de comunicación, a partir de la reformulación y ampliación de las ideas apuntadas por Redero y García (1991-1992):

1. Crisis económica general: desde 1973, el mundo occidental sufre una crisis general, conocida como la crisis del petróleo ya que es debida al aumento del precio del crudo. Este contexto económico será especialmente difícil para España, no solo porque el Estado tenía una economía débil (consecuencia de casi 40 años de autarquía) sino porque a la crisis general se le sumará la inestabilidad política y social en el Estado (con la consiguiente desconfianza en el sector económico que esto provoca) que genera el fin del régimen y el inicio de la transición.

2. Reconversión tecnológica: la irrupción de novedades tecnológicas tanto en las imprentas como las redacciones, con la llegada de los primeros ordenadores,

${ }^{6}$ El Periódico de Catalunya aparece el 28 de octubre de 1978

${ }^{7}$ El Sport saca el primer número el día 3 de noviembre de 1979. 
requerirán de una fuerte reconversión que no todos los medios tendrán capacidad económica para llevar a cabo.

3. Empresas desfasadas: incluso en los grandes medios (como La Vanguardia española, propiedad de una empresa familiar) imperará un sistema empresarial no profesionalizado, con unas estructuras desfasadas e impropias de una economía de mercado de finales del siglo XX y unas plantillas sobredimensionadas que dificultaban la viabilidad económica de la compañía (Barrera, 1995b: 460).

4. Descenso de la publicidad debido a la crisis económica. A la vez que bajan los ingresos publicitarios, habrá más empresas periodísticas que querrán entrar en el reparto debido a la aparición de nuevas publicaciones y, sobre todo, de la consolidación de nuevos medios más masivos como la radio y la televisión. Las empresas publicitarias preferirán concentrar los recursos en anuncios en medios de mayor difusión.

5. Ayudas públicas exiguas: A las dificultades propias que conlleva por sí mismo el hecho de editar un rotativo, se añadía la paradoja de que, aunque los medios de comunicación eran pieza fundamental en la construcción de la democracia, las ayudas del Estado hacia estas empresas fueron exiguas ${ }^{8}$ (Pascual, 1995: 517), y más si tenemos en cuenta que estas ayudas hay que verlas como una colaboración de la propia sociedad para dotarse así mismo de la prensa que necesita. Con la democracia, esta situación se corrigió y tanto los medios públicos como los privados optaron a subvención por parte del gobierno del Estado primero (Blasco Gil, 2008: 95-103) y a través de las Comunidades Autónomas después cuando las directivas europeas así lo exigieron (De la Quintana y Oliva, 2012: 106).

6. Lógica capitalista de mercado y cambio de modelo de negocio: Necesidad de que las empresas periodísticas se adecuen urgentemente a la realidad de mercado. El advenimiento de las libertades provoca la aparición de una oferta informativa que será muy superior a la demanda. Las cabeceras no podrán hacer frente a los cambios de imagen y de estilo imprescindibles en el contexto de una fuerte competencia. Todo ello en un contexto en el que, por culpa del franquismo, las empresas periodísticas no se habían podido desarrollar. Habrá, por tanto, que comenzar a estructurar proyectos empresariales sólidos.

En la actualidad, aunque los motivos son distintos, la crisis también se explica por este cambio del modelo de negocio provocado por la irrupción de internet

${ }^{8}$ Redero y Garcia (1991) apuntan, incluso, que la desaparición de muchas cabeceras se debió a la 'desidia' de los poderes públicos para protegerlas. 
y de la percepción de buena parte de la ciudadanía que la información no debe pagarse. Muchos de los fracasos de las empresas periodísticas entre 2008 y 2013 se explican por esta imposibilidad de encontrar un nuevo modelo de negocio que se adapte a esta situación al no encontrar una forma viable para hacer rentable los proyectos online (Casero, 2010: 595). La difusión de la prensa en España sigue cayendo desde 2008, pasando de casi 4.5 millones de ejemplares diarios a 3.5 millones.

7. Incremento del precio de los diarios: la situación de crisis, el descenso de ventas pero, sobre todo, el aumento de precio del papel prensa (el más caro de Europa) hará que el precio del ejemplar se triplique entre 1974 y 1978, pasando de 6 a 18 pesetas, en un incremento muy superior al aumento del coste de la vida.

8. Pérdida del 'privilegio' ideológico: La exclusividad (no solicitada ni deseada) de que muchas de las publicaciones antifranquistas habían disfrutado durante los últimos años de dictadura e inicio de la transición en la difusión de un discurso alternativo que entroncaba con los deseos de libertad de buena parte de la población, desaparece con la consolidación de la democracia.

9. Desgaste de imagen: A partir de las elecciones legislativas de junio de 1977 (las primeras democráticas), y durante todo 1978 , se empezará a vivir un proceso de desencanto político (narrado y alertado por los propios medios) que también afectará a aquellos que más habían significado y vinculado con la clase política, como eran los periodistas y los medios de comunicación. La prensa experimentará en carne propia el desgaste que afectará a instituciones y partidos.

\subsubsection{Cambios periodísticos}

La transición en la prensa (como se puede observar con el análisis de la prensa de Barcelona) no se vivió solo a nivel ideológico o empresarial. También las páginas de los periódicos fueron cambiando para adaptarse a los nuevos ritmos informativos, para tratar de ser atractivas a los ojos de los ciudadanos (para atraer nuevos lectores sin perder los que ya tenía), en definitiva, para hacer un producto competitivo que respondiera de la mejor manera posible a los nuevos retos que marcaba la economía de mercado.

A través del análisis de contenido realizado sobre los periódicos de Barcelona durante la transición, se observa cómo las páginas sin secciones fijas y establecidas, estructuradas de forma irregular, con niveles de lectura poco diferenciados y con grandes manchas de gris que a menudo recordaban más un libro que un periódico, irán dejando paso a periódicos más estructurados, con secciones claras y fijas, textos delimitados y más breves, más niveles de lectura (titulares, subtítulos y / o ante título, entradillas, destacados ...) y la información más fragmentada. Además, se consolidará la presencia 
de expertos opinadores (politólogos, profesores, sociólogos,...) a las páginas de los periódicos para aportar su visión sobre diferentes aspectos de la actualidad, sobre todo política.

Estos cambios, afectarán de forma clara las nuevas propuestas que irán surgiendo (EI País, Catalunya Express o El Periódico de Catalunya son dos rotativos que ya no tendrán el diseño como una cuestión secundaria sino que serán diarios muy pensados y que beberán de las influencias estilísticas de la prensa moderna europea y anglosajona), mientras que los periódicos ya existentes o bien se irán adaptando progresivamente (es el caso de $A B C$ o La Vanguardia que, a pesar de incorporar cambios en el aspecto de sus páginas, hasta 1989 no hará un rediseño definitivo) o bien ya no tendrán capacidad para adecuarse a los nuevos tiempos (situación que experimentarán, por ejemplo, periódicos como El Noticiero Universal, Solidaridad Nacional o La Prensa).

\subsubsection{Cambio de función: agente político}

El análisis de contenido de la prensa de Barcelona permite demostrar cómo entre 1975 y 1978, indudablemente la prensa se convertirá en el espacio a través del cual las élites políticas de oposición democrática tomarán contacto con la ciudadanía. Con la asunción de este rol de actor en el proceso político, la prensa contribuirá, en palabras del historiador Carlos Barrera (1995: 451), "indudablemente no tanto quizás a erosionar el régimen (no hay que olvidar que Franco murió en la cama) sino a preparar y difundir anticipadamente las ideas que hicieron posible luego la transición". Un rol político que no ha dejado de ejercer durante toda la democracia.

El periodismo escrito tendrá una influencia relevante en estos primeros compases de la transición, sobre todo durante el período crítico que transcurre desde la muerte de Franco hasta la aprobación de la Constitución (Guillamet, 2001: 269-274). Esta es la visión del historiador Pedro Pascual:

[El] grupo prensa fue el que más arriesgó desde el primer momento y el que más se jugó el tipo en la primera mitad del año 1976, mucho más que cualquier otro. Decir que la prensa, y en general los medios informativos, tuvo durante toda la transición política una participación tan decisiva como determinante en la llegada, afianzamiento y consolidación de la democracia en España es simplemente recordar lo que está en la mente de todos (1995: 517-518).

En este mismo sentido se expresa Juan Luís Cebrián cuando afirma que "uno de los fenómenos más peculiares de la transición política española fue el papel que en la misma desempeñaron los medios de comunicación, y de manera muy especial la prensa" (1996: 36) no solo como cooperadora necesaria para alcanzar las libertades, sino también como nos decisivo en la consolidación de los cambios que se iban produciendo y en la superación, e incluso neutralización, de conflictos que iban apareciendo (Redero y García, 1991-1992 : 100-101). Tanto es así, que podemos 
entender la prensa como una de las causas que provocaron la no continuidad del régimen franquista y el triunfo de las tesis demócratas en convertirse en un auténtico cuarto poder (Carr, 2001: 232).

Esta implicación política de la prensa barcelonesa, catalana y española en el proceso histórico que se estaba viviendo, no fue un hecho aislado. Borrat sostiene que el diario de información general es un verdadero actor político de naturaleza colectiva, el ámbito de actuación es el de la influencia, no el de la conquista del poder institucional o la permanencia en él. El periódico influye así sobre el gobierno, pero también sobre los partidos políticos, los grupos de interés, los movimientos sociales y su audiencia (1989: 10) para convertirse en un componente esencial en el funcionamiento de un régimen de libertades y el constructor del espacio público (Redero y García, 1991 a 1992: 85). La prensa hace de altavoz de reivindicaciones pero a la vez se convierte en protagonista del evento político.

Los medios escritos contribuyeron en el terreno que les era más favorable: en el de construir y hacer suya la opinión pública. La participación ciudadana en la vida política se abrirá, después de los años de ostracismo franquista, y en ello tendrán mucho que ver los partidos políticos de oposición, los sindicatos, la sociedad civil pero también, y de forma relevante, los medios de comunicación que se conjuran para difundir los valores del nuevo sistema (Redero y García, 1991-92: 97, 103). Los medios tomarán partido y asumirán la responsabilidad de contribuir a construir el cambio de régimen (Barrera, 1995: 463). Será por este motivo que en la prensa de ese momento la salvaguarda de las libertades prevalecerá, en algunas ocasiones, por encima de principios periodísticos como la objetividad o la neutralidad.

Entendiendo, tal y como sostiene Habermas (1987: 528), que la comunicación es una pieza clave en la conformación de las sociedades actuales, se puede hablar de la contribución de la prensa en conseguir que los temas de debate político fueran precisamente los que ellos mismos ponían sobre la mesa. Un papel, el de la prensa, que a juicio de Seoane y Saiz fue excesivo en conllevó una fuerte implicación política e ideológica de los periodistas que sentirse protagonistas del cambio, y no simples narradores de los acontecimientos que sucedían (2007: 299).

Este rol que irán asumiendo los medios y los profesionales marcará una extrema conexión y también interdependencia entre el poder político y el 'poder' periodístico. Este hecho también explica que durante los años de la transición ( $y$ en algunos casos incluso antes), se vaya tejiendo una estrecha complicidad entre bastantes políticos y periodistas; relación que, a pesar de tener aspectos positivos por lo que significaba de reforzamiento del sistema democrático, también tendrá de negativos que podrán repercutir en la propia crisis que afectará a las empresas periodísticas a finales de los 70: esta vinculación contribuirá a menudo al elitismo informativo de los medios y, por otra parte, en la intromisión o creación de periódicos excesivamente ideologizados o, 
incluso, claramente partidistas (Gómez Mompart, 1999: 212). Este vínculo en exceso entre periodistas y políticos explicará, en parte, que el desprestigio que irá adquiriendo la clase política ante la ciudadanía ya desde la década de los 80 , se acabe extendiendo también hacia los profesionales de la información.

\section{Conclusiones}

El estudio a fondo de la prensa de Barcelona durante la transición democrática permite trazar un mapa bastante claro de la crisis y cambios que el sector experimentó. Una crisis ideológica (acompañada de cambios legales y políticos); económica (global, del sector y publicitaria) y empresarial; y de reconversión tecnológica.

Estas crisis afectaron de forma preeminente la industria periodística y además estuvieron acompañadas de un cambio de modelo informativo $y$, también, de reconversión tecnológica. El proceso de adaptación a la nueva realidad tecnológica fue letal para muchos medios que no supieron adaptarse. Toda esta situación se tradujo en despidos de profesionales y cierre de medios, compensada con la creación de nuevos puestos de trabajo en nuevos medios que empezaban; un dinamismo del mercado que para nada se encuentra en la actualidad.

Sin duda, y como afirma Almirón (2009), la crisis actual (iniciada en 2008) de los medios solo es comparable con la que vivió el sector a partir de 1975. Y es que en la actualidad, la situación de crisis del sector periodístico también se explica por razones económicas (global, del sector y publicitaria) y empresariales (con la necesidad de un nuevo modelo de negocio); de reconversión tecnológica; y de modelo informativo y de negocio. La situación actual presenta como agravante que todavía no se ha encontrado un modelo de negocio que pueda competir, de forma económicamente viable, con la difusión de información gratuita online.

La crisis del sector actual, pues, afecta la estructura misma del periodismo, de la difusión de la información y del modelo de negocio. Una crisis estructural (y no solo coyuntural como lo fue durante la transición). Este estudio a fondo de los cambios de la prensa durante la transición abre perspectivas de análisis comparativo con la situación de crisis contemporánea. Seguidamente se apuntan algunas líneas de investigación que, desde metodologías de análisis comparado, permitan establecer esta comparativa entre las distintas situaciones de crisis y cambio en el sector periodístico.

Así, en la situación actual, también encontramos cambios de índole económico/empresarial: entre 2008 y 2015 han desaparecido en España un total de 375 empresas periodísticas. Esta cifra corresponde a tres agencias de información, 38 
diarios, 58 periódicos digitales, 12 gratuitos, 11 radios, 39 televisiones y 214 revistas. Pero a su vez, se han creado en este mismo periodo un total de 458 medios, según el Informe Anual de la Profesión Periodística 2015. En este mismo informe se señala que "los periodistas españoles han puesto en marcha medio millar de proyectos, en su mayor parte informativos, impulsados por esa crisis, como consecuencia de la pérdida del puesto de trabajo o por la imposibilidad de acceder a un empleo, profesionales experimentados y recién licenciados se vieron obligados a desarrollar una labor como emprendedores en la que no tenían experiencia ni conocimientos" si bien, como señala el mismo informe, se trata de medios mayoritariamente con escasez de recursos y víctimas de la precariedad laboral, la principal preocupación de los periodistas españoles, según este mismo estudio.

En sólo cinco años (de 2007 a 2011), los periódicos españoles sufrieron unas pérdidas de beneficios del 88\%: de 232,9 millones a 28,1 millones de euros (AEDE, 2012). Como se comentaba anteriormente, esto se debe a la crisis financiera global y a la propia que sufre la prensa (Casero, 2010: 596). Esta situación, que no será ni mucho menos el aspecto principal de la crisis durante los años de la transición, adquiere un gran protagonismo en el contexto actual con unos medios que deben hacer frente a la irrupción de la web 2.0 y las redes sociales (Casero, López-Meri, 2015), con lo que conlleva también de predominio de la gratuidad en el acceso a la información en internet.

Si bien a inicios del siglo XXI las empresas periodísticas afrontan la crisis desde una posición de profesionalización, el cambio en el modelo de negocio fruto de la reconversión tecnológica y la necesidad de buscar nuevas fuentes de ingresos también provocarán una sacudida muy severa a las estructuras empresariales. Así, aunque el consumo de información ha aumentado (básicamente por internet), las empresas periodísticas no consiguen traducir este incremento en más ingresos que hagan rentables los negocios (Casero, 2010: 595). Claramente, y como se indicó en el inicio de este artículo, este es uno de los principales problemas al que ha tenido que hacer frente el sector de la comunicación desde 2008. En España, la publicidad se ha reducido un 40,4\% hasta 2010 (Infoadex, 2010). Y aunque a partir del 2014 esta caída frenó, en 2016 la inversión publicitaria en prensa siguió cabiendo un 5.8\% respecto el año anterior (Arce Media).

A la vez, la situación de asfixia financiera de las instituciones públicas, y la necesidad de reducir el gasto, ha llevado desde 2012 a una severa reducción de estas ayudas públicas $^{9}$.

\footnotetext{
${ }^{9}$ En el caso catalán, en 2013 se ha producido una reducción del 60\% de las ayudas públicas a medios de comunicación (Fernández y Blasco, 2014).
} 
Y lo que hace 40 años se podía llamar pérdida de privilegio ideológico, en la actualidad se puede comparar con la pérdida del privilegio de la información. Las redes sociales comentadas en otros apartados han provocado que no sólo los periodistas tengan acceso directo e inminente a las fuentes informativas sino que también los ciudadanos pueden acceder con facilidad a estos recursos hasta hace poco reservados a la prensa (Castells, 2001; López, Martínez y Abejón, 2010).

Como se apuntaba cuando se analizaban los cambios económicos y empresariales, la irrupción de internet y de la web 2.0 ha conllevado también cambios profundos en la forma de hacer periodismo en el siglo XXI. Ya no son solo los medios tradicionales los que difunden noticias sino que la información llega a los ciudadanos a través de múltiples canales. Ante esta situación, los editores han ido asumiendo, aunque de forma tardía, la necesidad de cambiar el modelo informativo para adaptarse a la convergencia digital con lo que han ido apareciendo nuevos proyectos periodísticos Online (Casero, 2010: 595). Un cambio de modelo informativo que, como se apuntaba, todavía no ha encontrado un modelo de negocio estable.

Ya sea por la percepción ciudadana de falta de objetividad, pluralismo y neutralidad de los medios (Alsius, 2010) - un comportamiento que podría considerarse adecuado durante la transición pero no ya en plena democracia - o por la excesiva politización y falta de independencia política de los mismos (Alsius, 2011), pero lo cierto es que este desprestigio de la prensa al final de la transición se repite en la actualidad: como constatan los datos esta del barómetro del Centro de Investigaciones Sociológicas (CIS) de España, los periodistas son los profesionales peor valorados, solo por delante de los jueces ${ }^{10}$.

Cambios económicos, empresariales, de reconversión tecnológica y de modelo periodístico como sucedió durante la transición. De hecho, la crisis económica de inicios del siglo XXI marca una cuarta ruptura en la evolución del periodismo en España, esta vez no vinculada a motivos políticos pero si económicos y con coincidencias en las consecuencias que conlleva esta ruptura respecto a las rupturas anteriores.

\footnotetext{
10 Los periodistas reciben una calificacion de 59,09 sobre 100 por parte de los ciudadanos encuestados por la encuesta del Centro de Investigaciones Sociológicas (CIS) del año 2013. Sólo superan, por poco, a la percepció de los jueves $(59,02)$.

Fuente: http://www.cis.es/cis/opencm/CA/1_encuestas/estudios/ver.jsp?estudio=13664
} 


\section{Referencias bibliográficas}

AEDE, Asociación de Editores de Diarios de España (2013): Libro blanco de la prensa diaria 2013, Madrid, AEDE.

ALMIRON, N. (2009): "Grupos privados propietarios de medios de comunicación en España: principales datos estructurales y financieros" en Communication and Society/Comunicación y Sociedad, v. XXII, no 1, 2009, pp. 243-263.

ALSIUS, S. (ed.) (2010): The ethical values of journalists, Barcelona, Lexikon.

ALSIUS, S. Y SALGADO, F. (eds) (2011): La ética informativa vista por los ciudadanos, Barcelona, Editorial UOC.

BARRERA, C. (1995 a): Periodismo y franquismo. De la censura a la obertura, Barcelona, Ediciones Internacionales Universitarias Eiunsa.

- (1995 b): "Factores de cambio en el periodismo de la Transición”, en Tusell, J. y Soto, A., Historia de la transición y consolidación democrática en España, v. 2, Madrid, UNDE y UAM.

BLASCO GIL, J. J. (2008): “Las ayudas públicas a la prensa de las Comunidades Autónomas españolas. Tipología, cuantía de las subvenciones y sistemas de adjudicación" en Telos: Cuadernos de Comunicación e Innovación, no 75, 2008, pp. 95-103.

BORRAT, H. (1989): El periódico, actor político, Barcelona, Editorial Gustavo Gili.

CARR, R. (2001): España: de la Restauración a la democracia, 1875-1980, Barcelona, Ariel Historia.

CASANOVA, J. (1994): "Las enseñanzas de la transición democrática en España”, en Redero, M. (ed.), La Transición a la democracia en España. Revista Ayer, oㅡ 15, 1994.

CASERO-RIPOLLÉS, A. (2010): "Prensa en internet: nuevos modelos de negocio en el escenario de la convergencia" en El profesional de la información, v. 19, no6, noviembre-diciembre 2010, pp. 595-601.

CASERO-RIPOLLÉS, A. Y LÓPEZ-MERI, A. (2015): “Redes sociales, periodismo de datos y democracia monitorizada", en Campos Freire, F. y Rúas Araújo, J. (eds.), Las redes sociales digitales en el ecosistema mediático, La Laguna, Sociedad Latina de Comunicación Social, pp. 96-113

CASTELLS, M. (2001): “Internet y la sociedad red" en La factoría, no14-15, 2001. 
CEBRIÁN, J.L. (1996): "El País, un intelectual colectivo de la transición", en Juliá, S; Pradera, J; y Prieto, J., Memoria de la transición, Madrid, Taurus.

COSTA, P.O. (1989): "La premsa de la dècada", en Alemany, J., El periodisme en temps difícils, l'Escola del CIC (1964-1974), Barcelona, Diputació de Barcelona y Col·legi de Periodistes de Catalunya.

FERNÁNDEZ, I. y BLASCO, JJ (2014): "Press subsidy policies in Spain in the context of financial crisis (2008-2012): An analysis of the Catalan case" en European Journal of Communication, v. 29, no2, pp.171-187.

GÓMEZ MOMPART, J.L. (1994): La Gènesi de la premsa de masses a Catalunya, Barcelona, Editorial Pòrtic.

GUILLAMET, J. (1994): Història de la premsa, la ràdio i la televisió a Catalunya. 16411991, Barcelona, Edicions La Campana

- (2001): "El periodismo en la transición del franquismo a la democracia. Factores políticos, económicos y profesionales", en Company, A.; Pons, J.; y Serra, S., V Encontre de l'Associació d'Historiadors de la Comunicació, Palma, Universitat de les Illes Balears.

- (2003a): Els orígens de la premsa a Catalunya. Catàleg de periòdics antics (1641-1833), Barcelona, Ajuntament de Barcelona.

- (2003b): Història del periodisme : notícies, periodistes i mitjans de comunicació, Barcelona, Universitat Autònoma de Barcelona.

HABERMAS, J. (1987): Teoría de la acción comunicativa, v.2, Madrid, Taurus.

KAYSER, J. (1974): El diario francés. Barcelona, ATE.

LÓPEZ, M; MARTíNEZ, Y; ABEJÓN, P (2010): “Nuevos entornos, nuevas demandas, nuevos periodistas" en Estudios sobre el mensaje periodístico, n16, 2010, pp. 7994.

MAURI-RIOS, Marcel. (2010). Funció i evolució de la premsa de Barcelona duant la transició democràtica (1975-1978), Universitat Pompeu Fabra, Tesis doctoral.

PASCUAL, P. (1995). "La Prensa construyó la democracia”, en Tusell, J. Y Soto, A., Historia de la transición y consolidación democrática en España, v.2, Madrid, UNED y UAM.

QUINTANA, A. y OLIVA, C. (2012): "Subvenciones a la prensa en España: sinergías políticas y empresariales en Andalucía, Cataluña, Galicia y País Vasco" en Miguel Hernández Communication Journal, v.3, 2012, pp. 101-120. 
REDERO, M. y GARCÍA, G. (1991-1992): “Prensa y opiníon pública en la transición política española", en AA.VV, Anales de la Universidad de Alicante. Alicante, Universitat d'Alacant.

REIG, R. (2015): Crisis del sistema, crisis del periodismo, Barcelona, Gedisa.

SEOANE, M. y SÁIZ, M. (2007): Cuatro siglos de periodismo en España. De los avisos a los periódicos digitales, Madrid, Alianza Editorial.

TUSELL, J. (2005): Dictadura franquista y democracia, 1939-2004. Historia de España, XIV, Barcelona, Crítica. 